\title{
NOTICIA SOBRE UNOS FRAGMENTOS DESCONOCIDOS DE LA OBRA DE OVIDIO
}

Fragments on Ovid's Amores and Metamorphoses from the Stadtbibliothek at Trier are reported for the first time.

Eulogio Baeza solicitó a la Biblioteca Pública de Trier, la antigua Augusta Treverorum, una copia en microfilme del Fragmentum Trevirense, citado, entre otros, por R. Ehwald, S. G. Owen (en su edición oxoniense de 1915) y G. Luck en su edición y comentario de Tristia, y por el resumen de la historia del texto de Tristia de R. J. Tarrant ' . Cuando se recibió el microfilme, nos dimos cuenta de que los responsables de la Biblioteca enviaron, en lugar del fragmento citado de Tristia, otro fragmento pequeño, casi ilegible, que se decía que pertenecía a Ars amatoria. Los microfilmes eran dificiles de seguir, pero no nos cabía ninguna duda de que allí no se leía nada de Ars, sino de Amores y Metamorfosis. Y ya se sabe que, cuando un microfilme no sirve para leer un texto, lo mejor que uno puede hacer es trasladarse al sitio donde se encuentre el manuscrito que sea.

En el verano de 1994 tuvimos la oportunidad ${ }^{2}$ de trabajar en la misma Stadtbibliothek de Trier, muy cerca de la Basílica de Constantino y a la derecha de un precioso parque que llega justamente hasta las restauradas Termas del mismo Constantino. Nada más llegar a la Biblioteca, el Dr. Nolden, a quien agradecemos efusivamente su ayuda, nos tenía ya preparada una subcarpeta con los fragmentos de Ovidio. Nuestra sorpresa fue mayúscula. En dicha subcarpeta el Sr. Schoemann, bibliotecario del siglo pasado, había reunido en 1868 los fragmentos que estaban ante nuestros ojos. Se trata de hojas sueltas de antiguos manuscritos que sirvieron de cubiertas para encuadernar libros incu-

En L. D. Reynolds, ed., Texts and Transmission, Oxford 1983, p. 283.

2 Debemos agradecer las ayudas prestadas para la realización de este viaje a la Universidad de Huelva, a la Dirección General de Universidades de la Junta de Andalucía y a la Dirección General de Investigación Científica y Técnica del Ministerio de Educación y Ciencia (PB92-0486). 
nables de temas religiosos. El fragmento de Tristia parece haber estado antes aparte, pues aparece incorporado al final del folio que escribió el bibliotecario indicando el contenido de los fragmentos.

Los seis fragmentos son los siguientes:

\section{Fragmentum I (Met. V 574-VI 55)}

Es una hoja de tamaño folio escrito a dos columnas y con amplias anotaciones marginales.

$r=\mathrm{V}$ 574-614 conticuere undae... praecedere longam

615-654 ante pedes umbram... mihi peruius aether

$\mathrm{v}=\mathrm{V}$ 655-VI 15 dona fero Cereris... dumeta Timoli

VI $16-55$ deseruere suas... secernit harundo

Fragmentum II (Met. VII 292-451)

$r$ = VII 292-331 membraque luxuriant... limina natae

332-371 ambierantque torum... Cycneia Tempe

$v=372-411$ quae subitus... radiosque micantes 412-451 obliquantem oculos... gauentum

Es una hoja de tamaño folio escrito a dos columnas y con amplias anotaciones marginales.

La hoja del anterior fragmento y ésta formaban las páginas de respeto y el lomo que sirvieron para encuadernar seguramente uno de los muchos incunables que, procedentes de la Iglesia jesuítica de San Matías, han venido a parar a una sala aislada del sótano de la Biblioteca de Trier.

Fragmentum III (Met. VII 218-293, 456-539, 709-788, VIII 414-493)

Se compone de cuatro folios, distribuidos así:
f. $1^{r}=$ VII 218-258 nec frustra uolucrum... circuit aras
f. $1^{v}=$ VII 259-293 multifidasque faces... miracula monstri
f. $2^{r}=$ VII $456-497$ bella parat Minos... pignora formae
f. $2^{v}=$ VII 498-539 ingreditur ramumque... recumbere sulcos
f. $3^{\mathrm{r}}=$ VII 709-749 sacra tori coitusque... succumbere culpae
f. $3^{v}=$ VII $750-788$ me quoque muneribus... addere tempto
f. $4^{r}=$ VIII $414-453$ at manus Oenidae... pollice nentes
f. $4^{v}=$ VIII $453-493$ staminaque impresso... displicet auctor

Los folios $1^{\mathrm{v}}, 2^{\mathrm{r}}$ y $4^{\mathrm{v}}$ son ilegibles casi en sus tres cuartas partes por rasuras. Presenta abundantes notas marginales y supraversales. 
Fragmentum IV (Met. X 96-183, 551-593, 596-638)

Se compone de cuatro folios, distribuidos así:

f. $1^{r}=X$ 96-140 amnicolaeque simul... cacumine caelum

f. $1^{v}=X$ 141-183 ingemuit tristisque... properabat at illum

f. $2^{r}=X$ 551-593 impetus est fuluis... genualia limbo

f. $2^{v}=X 596-638$ candida purpureum... populusque paterque

El folio $2^{\mathrm{r}}$ contiene múltiples rasuras que dificultan mucho su lectura. Presenta abundantes notas marginales y supraversales.

Fragmentum $V(A m$. III $3,12-48 ; 4,1.48 ; 6,1-10)$

Se conserva un solo folio de tamaño pequeño, con abundantes notas marginales y supraversales.

El folio se distribuye así:

$\mathrm{r}=$ III 3, 12-III 4, 8, sed uu. 9-12 desiderantur

$v=$ III 4, 13-III 6, 10 (omittit III 5)

Sus lecturas más significativas son:

III 3, 13 tuos; 17 ; 44 fallat

III 4, 16 lapsa; 17 cupita semperque negata; 21 thalamo, perenni; 22 credita; 33 non

Podríamos fecharlo en los siglos XII-XIII, porque sus lecturas coinciden con la familia non $\alpha$, que forman los recentiores ${ }^{3}$.

Fragmentum VI (Tristia I 11, 1-31, I 11, 33-2, 21, IV 4, 35-65, IV 4, 67-IV 5, 9)

Este fragmento, colacionado por primera vez por Ehwald en una tarde de excursión de 1892, se compone de dos folios de pergamino de $200 \times 150 \mathrm{~mm}$., procedentes de un $\mathrm{ms}$. del $\mathrm{s}$. $\mathrm{x}$ escrito en letras minúsculas. Estas dos hojas sirvieron de cubierta para otro ms., probablemente de la Biblia, de la que hay muchas copias en la Stadtbibliothek de Trier. Ambos folios están unidos mediante una trenza, que oculta los comienzos o finales de verso, conservados perfectamente.

Desde luego, parece que su origen no es el mismo de los otros cinco fragmentos, pues Schoemann no lo detectó en 1868. En la relación que aparece en

${ }^{3}$ Cf. E. J. Kenney, «The Manuscript Tradition of Ovid's Amores, Ars amatoria, and Remedia amoris", CQ 12, 1962, p. 9; The Classical Text, Berkeley 1974, p. 134. 
un folio de la subcarpeta está añadido a bolígrafo (por consiguiente no antes de la II Guerra Mundial) por alguien, que además sólo da $T r$. IV 4 y 5 (f. 2) y no el resto I 11, 1-II 21 que sería el $\mathrm{f}$. 1 .

El fragmento se distribuye de la siguiente forma:
f. $1^{r}=1$
$11,1-31$ (el v. 32 está perdido por rotura del ms.)
f. $1^{\mathrm{v}}=\mathrm{I}$ $11,33-1121$ (el v. 22 está perdido por rotura del ms.)
f. $2^{r}=I V$
4, 35-64 (los vv. 65-66 están perdidos por rotura del ms.)
f. $2^{\mathrm{v}}=\mathrm{IV}$
$4,67-5,9$.

En el $\mathrm{f} . \mathrm{I}^{\mathrm{r}}$ es difícil leer los finales de verso. Realmente, es prácticamente ilegible el texto ya por el desgaste producido por el uso o ya por los raspados que ha sufrido.

El único fragmento utilizado hasta ahora ha sido el de Tristia, que curiosamente aparece como añadido en la subcarpeta de Trier. La colación fue realizada por R. Ehwald en Ad historiam carminum Ovidianorum recensionemque symbolae, Gotha 1892, pp. 3-5. El fragmentum Trevirense ha sido utilizado después solamente en las ediciones de Tristia, como en las de Owen, Luck y André.

Munk Olsen cita el fragmento ${ }^{4}$ señalado por Ehwald para Tristia, además de otros restos de Metamorfosis y Ars procedentes de un Florilegium prosodiacum ${ }^{5}$, ff. $42^{\mathrm{v}}-44^{\mathrm{v}}$. Su fuente de información ha sido la disertación de Klug ${ }^{6}$, quien ofrece las lecturas de las Metamorfosis y de Ars en pp. 51-54. Sin embargo, no dice una palabra de los fragmentos I-V para Metamorfosis y Amores, de los que damos noticia aquí por primera vez.

\section{A. Ramirez de Verger EULOGIO F. BAEZA}

\footnotetext{
${ }^{4}$ B. Munk Olsen, L'étude des auteurs classiques latins aux XI et XII siècles, París 1985 , p. 166.

5 B. Munk Olsen, «Les clasiques latins dans les Florilèges médiévaux antérieurs au XIII siècle», RHT 9, 1979, pp. 70-71.

${ }^{6}$ E. Klug, De florilegiis codicis Monacensis 6292 et codicis Trevirensis 1092, Gryphiae 1913, pp. 17-19, 51-54 y 63.
} 


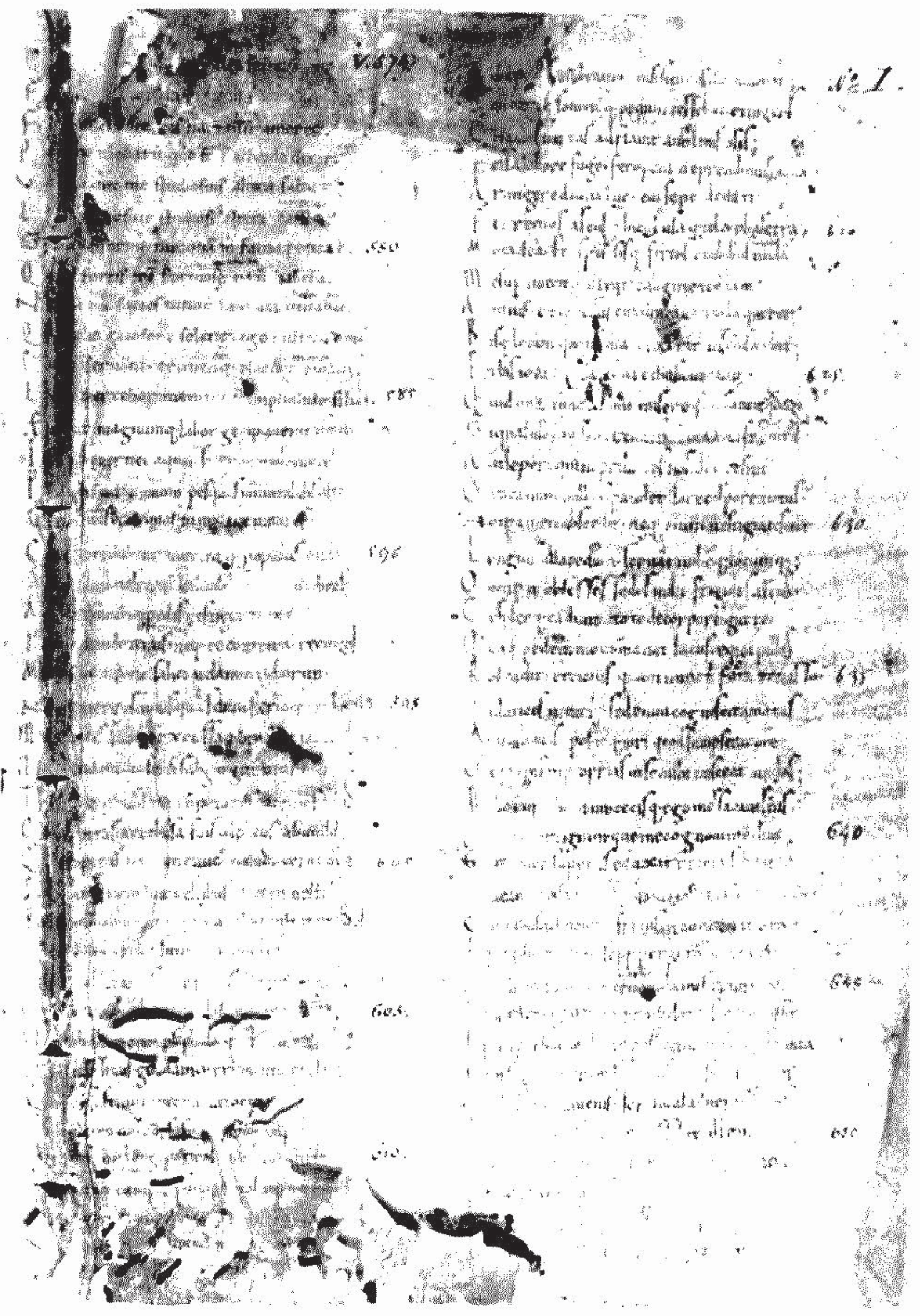

FRAGMLNTWM I

(c) Consejo Superior de Investigaciones Científicâs

http://emerita.revistas.csic.es

Licencia Creative Commons 3.0 España (by-nc) 


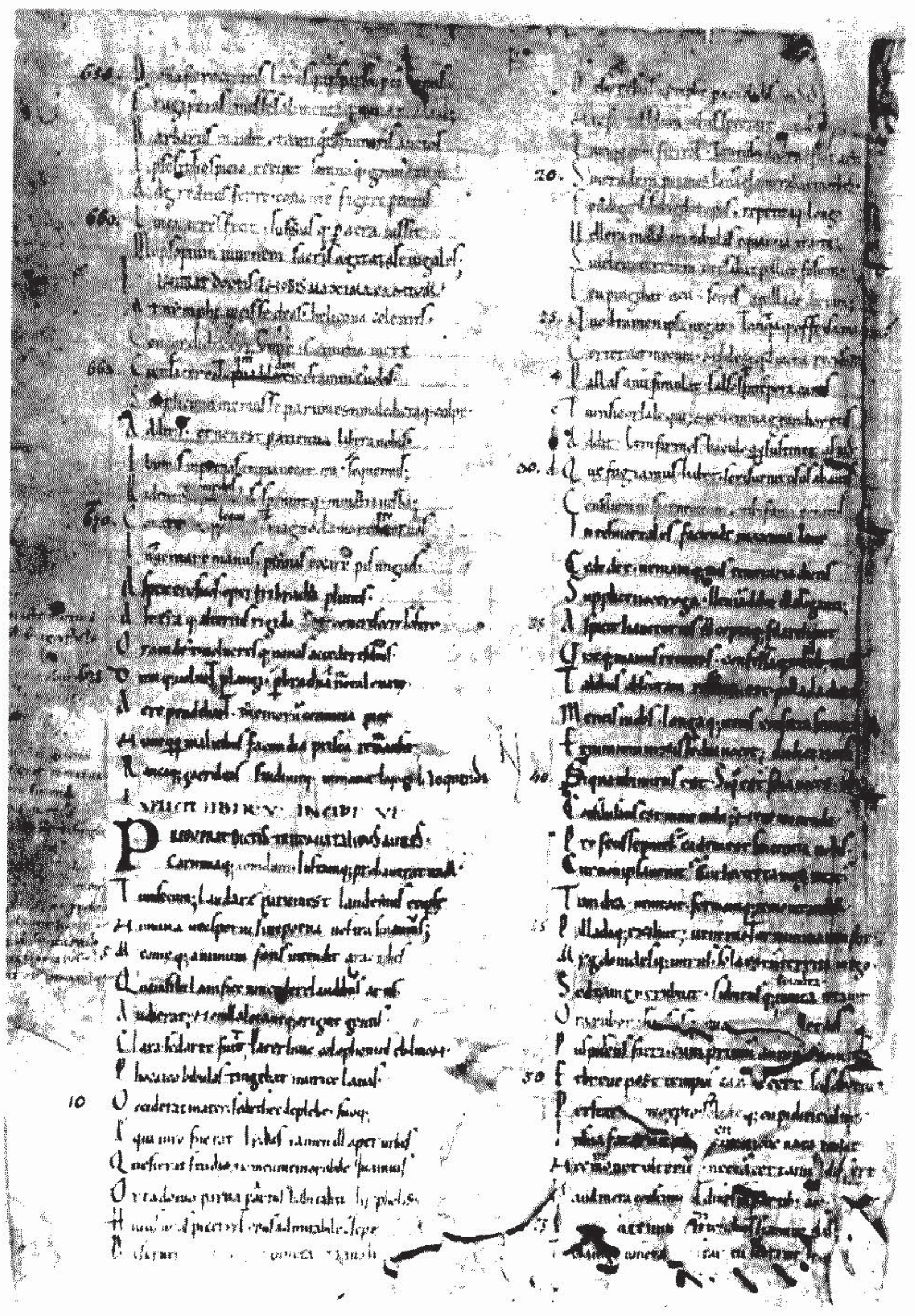




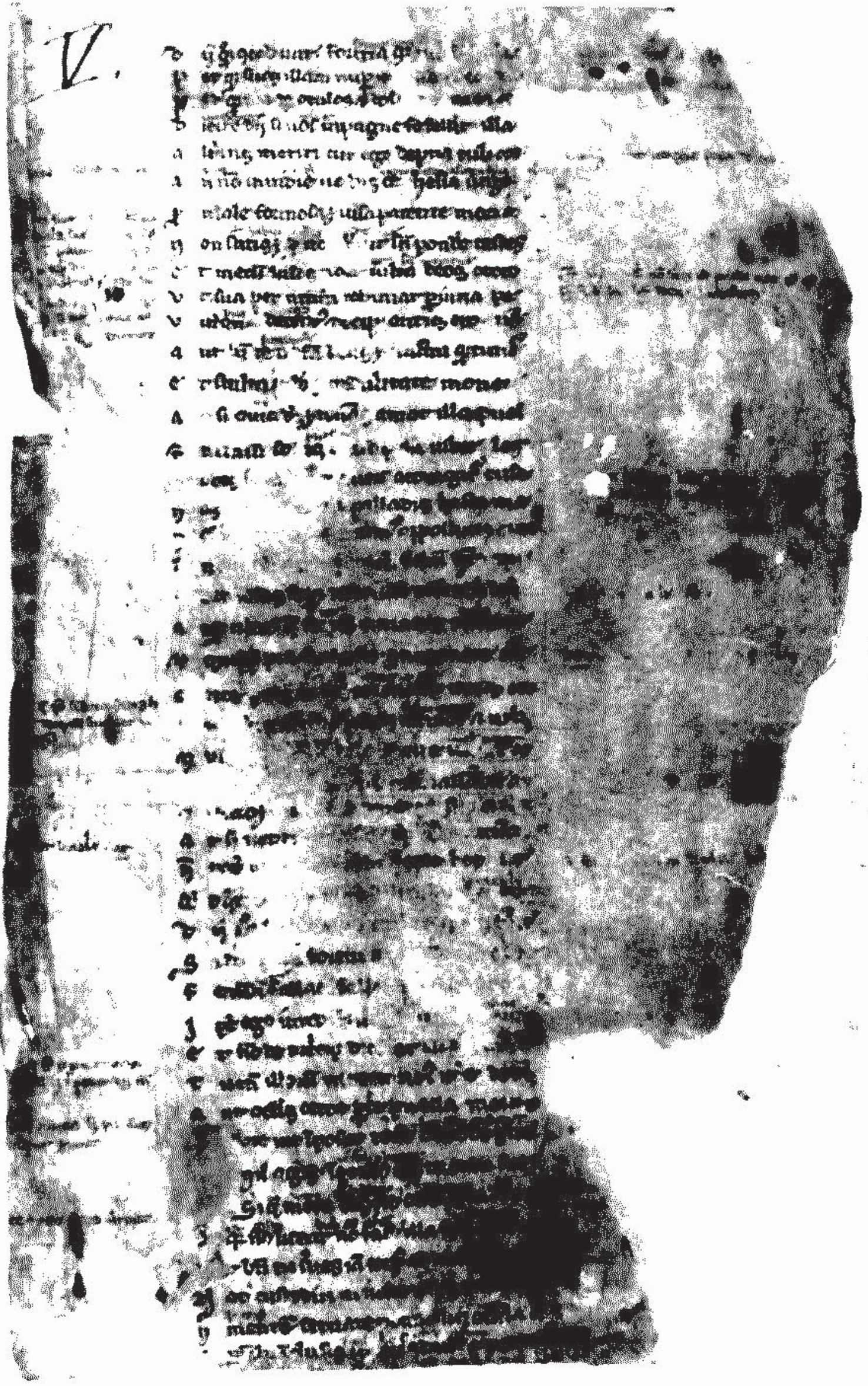

Fragmentum $V$

(c) Consejo Superior de Investigaciones Científicas 


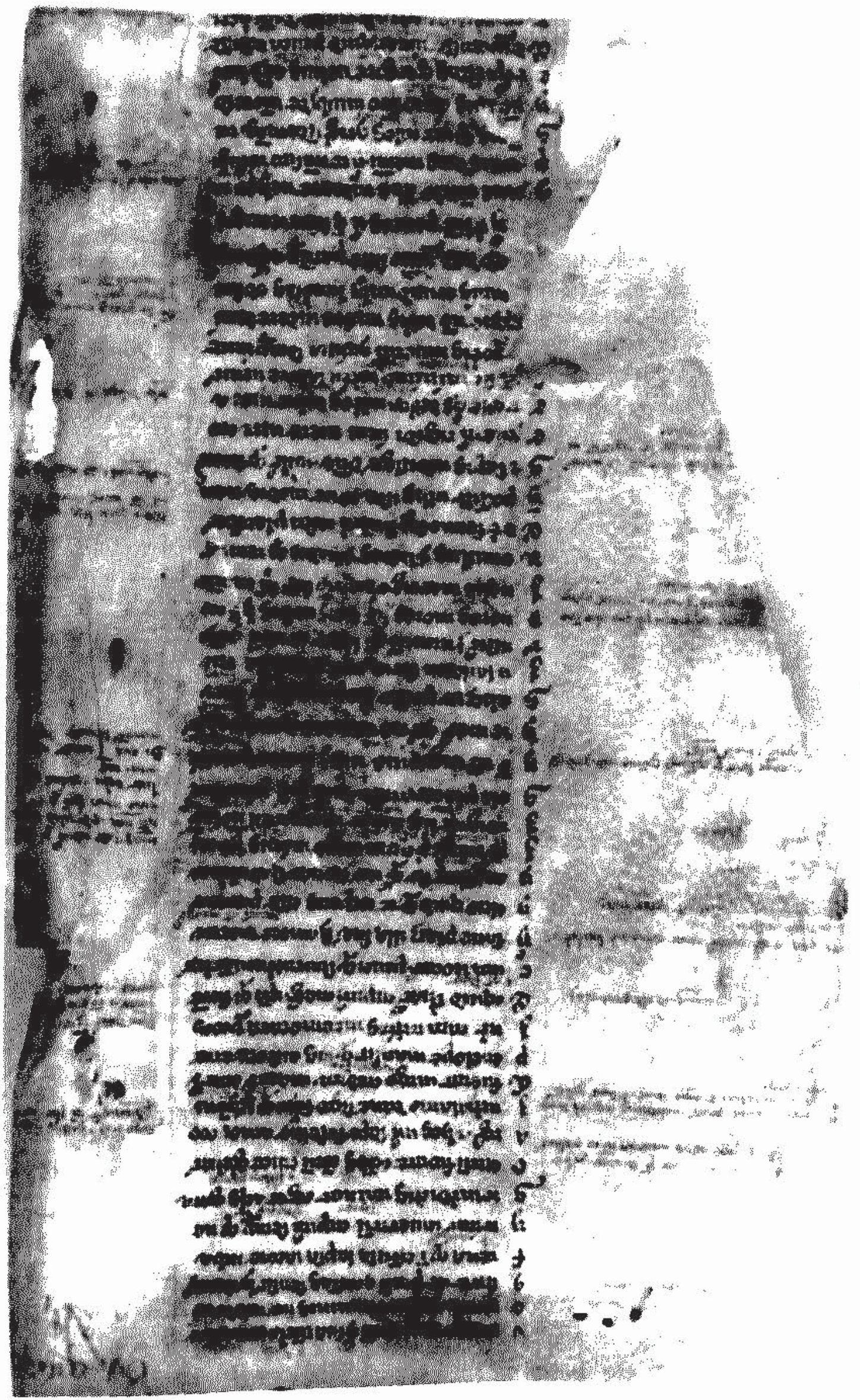

